\title{
4 Praxisinterne Behandlungspfade: Was bringen sie den Ärzten, Fachärzten und Psychotherapeuten?
}

Julia Bellabarba

Was sind praxisinterne Behandlungspfade? Der praxisinterne Behandlungspfad (oder diagnostische Pfad) ist eine strukturierte Beschreibung (bzw. Darstellung) eines (therapeutischen oder diagnostischen) Standardprozesses, der alle spezifischen organisatorischen und medizinischen Leistungen der Praxis abbildet. Wie kann der strukturierte Ablauf der Behandlung konkret aussehen? Abbildung 13 stellt einen Behandlungspfad zur Notfallversorgung dar. Er wurde in einer Gemeinschaftspraxis für Allgemeinmedizin erstellt: Den Praxisinhabern und dem Team ermöglicht diese Darstellung, bei der Versorgung von Notfallpatienten festgelegte Standards der Versorgung umzusetzen.

Für den „normalen“ Praxisbedarf ist es in der Regel einfacher, wenn die Pfade nicht als Flussdiagramme (also Flowcharts) aufgezeichnet werden, sondern in tabellarischer Form als Text. Eine mindestens dreispaltige Tabelle enthält alle notwendigen Informationen: Was wird gemacht? Wer ist zuständig? Welche Unterlagen/Materialien gehören dazu? Tabelle 13 zeigt einen Teil eines Behandlungspfades für Patienten mit systemischem Lupus erythematodes (SLE) erstellt von einer Arbeitsgruppe der Deutschen Gesellschaft für Rheumatologie (und von der Autorin in tabellarischer Form wiedergegeben). 


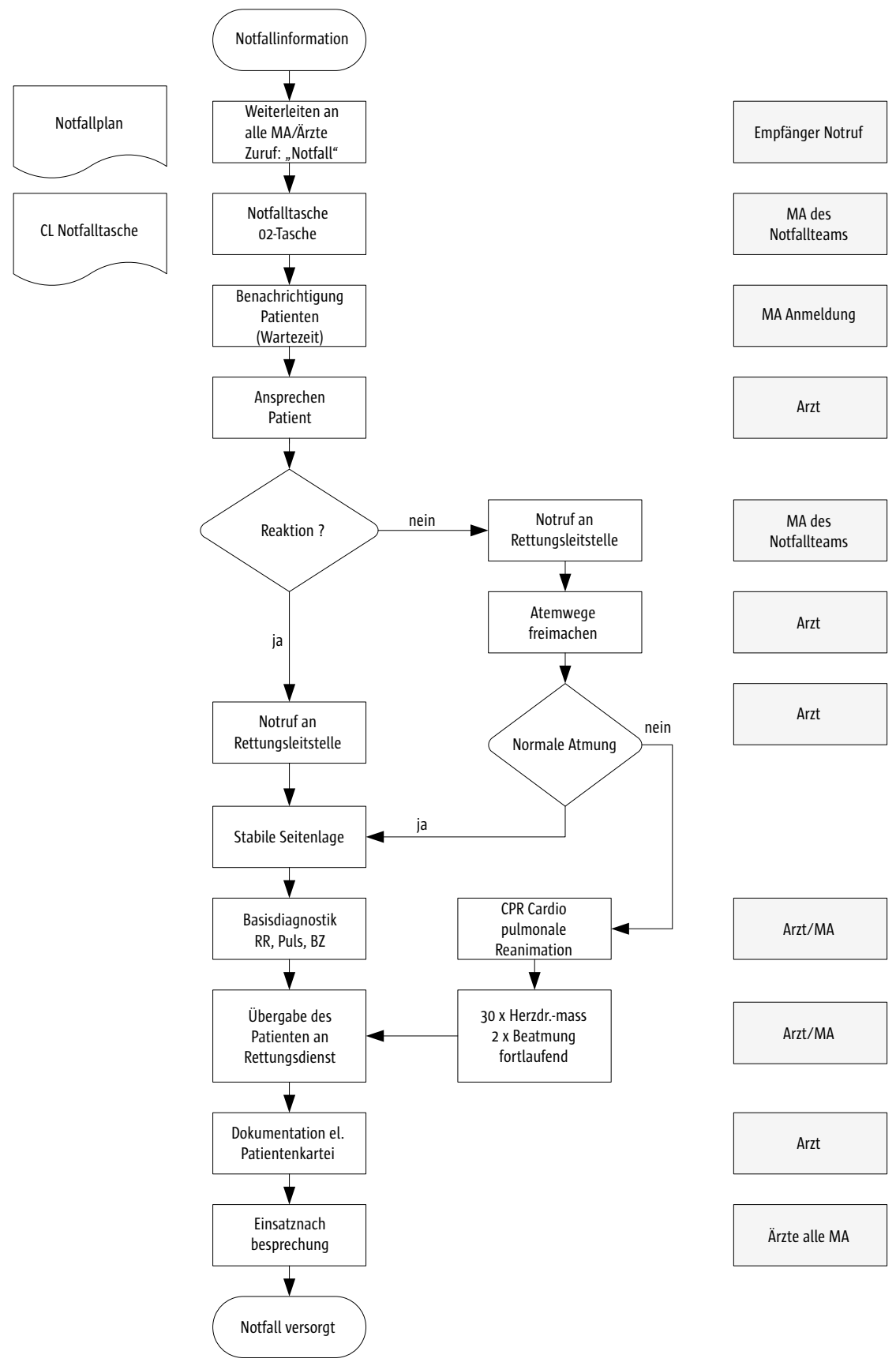

Abb. 13 Flowchart-Notfallmanagement 
4 Praxisinterne Behandlungspfade: Was bringen sie den Ärzten, Fachärzten und Psychotherapeuten?

Tab. 13 Behandlungspfad SLE tabellarisch

\begin{tabular}{lll} 
Was? & Wer? & Unterlagen? \\
\hline Vor jedem Arzt-Patientenkontakt: & & \\
$\begin{array}{l}\text { Blutdruckmessung, Urinbefund und Kontrolle des Vorliegens aller MFA } \\
\text { relevanten Laborbefunde. }\end{array}$ & Befunde \\
\hline
\end{tabular}

Zusätzlich werden Befunde erhoben, die nach Kontrollintervall

bzw. Anweisung des Arztes zu diesem Termin notwendig sind (ggf.

Vereinbarung Labortermin).

Die Patientenakte wird auf Vollständigkeit überprüft (z. B.

Patientenakte

Überprüfung, ob der neurologische Befund vorliegt). Wenn die

Unterlagen nicht vollständig sind, erläutert die Helferin dem

Patienten erneut die Wichtigkeit und kümmert sich ggf. um

Beschaffung fehlender Befunde.

Die Helferin bereitet die Akte vollständig vor und bringt diese in MFA/Arzt

das Untersuchungszimmer. Der Arzt informiert sich vor dem

Patientenkontakt kurz über aktuelle Werte.

Bei jedem Arzt-Patientenkontakt:

Aktualisierung der Anamnese. Zusätzlich werden bei jedem

Patienten folgende Fragen gestellt und dokumentiert:

Arzt

DOK ECLAM-

Bogen

Allgemeine Fragen zum Gesundheitszustand (Infektionen,

Befunde anderer Fachärzte, Besuch der Rheumaliga usw.)

Allgemeiner

Fragebogen der

Praxis

Wirksamkeit/Verträglichkeit/Nebenwirkungen der Therapie

Umsetzung der Therapie durch den Patienten

Eine symptombezogene körperliche Untersuchung wird durchgeführt:

Die Therapie wird geplant bzw. aktualisiert: Arzt Patientenakte

Therapie des SLE orientiert sich der klinischen Manifestation der

Erkrankung unter Berücksichtigung des Schweregrades.

Die medikamentöse Therapie stützt sich auf NSAR, Antimalaria-

mittel, Corticosteroide und Immunsuppressiva die entsprechend

ggf. Information

dem Schweregrad der Erkrankung eingesetzt werden.

zur Medikation

Der Arzt orientiert seine Therapieentscheidungen an den

Empfehlungen der Deutschen Gesellschaft für Rheumatologie.

Der Arzt trägt dafür Sorge, mögliche Organmanifestationen

ggf. Leitlinien

frühzeitig zu erkennen. Dazu sind eigene Untersuchungen oder

die Mitbehandlung von Fachkollegen geeignet.

Gemeinsam mit dem Patienten werden Therapievorschläge/

Entscheidungen/Änderungen besprochen.

ggf. Informations-

material für

Patienten 


\begin{tabular}{lll} 
Was? & Wer? & Unterlagen? \\
$\begin{array}{l}\text { Die daraus resultierenden notwendigen klinischen und labortech- } \\
\text { nischen Kontrolluntersuchungen werden geplant und durchge- }\end{array}$ & \\
führt: & \\
\hline $\begin{array}{l}\text { richten sich ebenfalls nach diesen Richtlinien und dem } \\
\text { Diagnose-/Therapieüberwachungsschema SLE (siehe IR }\end{array}$ & \\
Diagnostik). & \\
\hline $\begin{array}{l}\text { Auf dem Diagnostik-Laufzettel werden notwendig Untersuchun- } \\
\text { gen (sofort oder zum nächsten Termin durchzuführende }\end{array}$ & Diagnostik-Lauf- \\
Untersuchungen) vom Arzt dokumentiert und oben auf die Akte & \\
geheftet. Bei Bedarf erhält die Helferin eine kurze Erläuterung. & \\
\hline $\begin{array}{l}\text { Die Arzthelferin überwacht die Umsetzung der Maßnahmen } \\
\text { (Funktionsdiagnostik in der Regel sofort, Patienteninformation }\end{array}$ & MFA & \\
über Fachärzte, Überwachung, dass die zum nächsten Termine & \\
angewiesenen diagnostischen Maßnahmen veranlasst werden). & \\
(...) & \\
\hline
\end{tabular}

Was bringt das? Der praxisinterne Behandlungspfad beruht in der Regel auf evidenzbasierten Leitlinien, ist jedoch als eine individualisierte, auf die Gegebenheiten der Praxis bezogene Darstellung zu verstehen. Die QM-Richtlinie des GBA fordert die Dokumentation der praxisinternen Pfade nicht explizit, sondern weist auf die Verpflichtung hin, Behandlungsabläufe zu „strukturieren“. Darüber hinaus schreibt die Richtlinie vor, „als Instrumente eines einrichtungsinternen Qualitätsmanagements sind insbesondere zu nutzen: (...) Prozess- und Ablaufbeschreibungen“. Ich habe in Kapitel III.4 dargestellt, dass die wesentlichen Prozesse einer Praxis am Patienten stattfinden, es liegt also nahe, genau diese Prozesse $z u$ beschreiben und sie damit zu reflektieren, zu analisieren und zu verbessern. Viele niedergelassene Ärzte fragen: Was soll das? Ich weiß schließlich selber, was ich mache. Es ist in der Tat so, dass sich die Nützlichkeit praxisinterner Pfade umso mehr erschließt, desto komplexer (größer) die Praxis ist und desto komplexer (störungs- und fehleranfälliger) die zu beschreibenden Prozesse sind. Was also bringen praxisinterne Behandlungspfade der einzelnen Praxis?

Praxen, die eigene Pfade erstellt haben und von der Autorin dazu befragt wurden, haben die Vorteile so beschrieben:

- Die Erstellung des Pfades führt zu einer Reflexion (oder Diskussion im Team) über notwendige Standards der Behandlung.

- Praxisinterne Behandlungspfade ermöglicht es, zu reflektieren: Wie integriere(n) ich (wir) neues Zusatzwissen in die bewährten Behandlungsabläufe?

- Der Praxisleitung erleichtert der Pfad die Delegierbarkeit von Einzelleistungen.

- Der Pfad gibt allen Beteiligten im Team Sicherheit, besonders beim Einstieg neuer Mitarbeiter. 
4 Praxisinterne Behandlungspfade: Was bringen sie den Ärzten, Fachärzten und Psychotherapeuten?

- In der psychotherapeutischen Praxis: Das Therapiemanual bildet die idiosynkratischen Vorgehensweise des Psychotherapeuten nicht ab. Der Pfad hat den Vorteil: Man definiert, was man real in der Psychotherapie tut und überprüft die Wirksamkeit.

- Behandlungspfade schaffen die Transparenz, die im Rahmen von Honorarverhandlungen nötig ist.

- Unmittelbar sinnvoll (auch für die einzelne Praxis) erscheinen Pfade immer dann, wenn sie sektorenübergreifende, integrierte Prozesse abbilden (vor allem, weil die Erstellung sektorenübergreifender Pfade einen intensiven Austausch aller Beteiligten voraussetzt, der meist wichtiger ist als das Ergebnis „Pfad“).

Überprüfung derWirksamkeit der Behandlung: die Erhebung von Prozessindikatoren und Ergebnissen ist eines der wichtigsten Instrumente für die Qualitätsentwicklung der Organisation. Wir haben sie im Kapitel III. 4 und III. 5 beschrieben. Die Praxis, die eigene Behandlungspfade erstellt, sollte also immer die Überprüfung (CHECK im PDCA- oder Deming-Kreis) des Behandlungserfolges in den dokumentierten Pfad integrieren. Indem die Evaluation der eigenen Behandlungsbemühungen als ein Teil Ihres praxisinternen Behandlungspfad beschrieben (und durchgeführt) wird, enthält der Pfad eben genau die Informationen „die einen Unterschied machen“ und ist damit eine wirklich gewinnbringende Informationsquelle für die Verbesserung Ihrer Behandlungsqualität.

Sie finden hier ein Beispiel aus dem hervorgeht, wie die Erhebung von Indikatoren innerhalb eines Behandlungspfades verbindlich festgelegt werden kann.

\section{Praxisbeispiel: Qualitätssicherung im Rahmen eines Behandlungspfades für Patienten mit „systemischem Lupus erythematodes (SLE)“ erstellt von einer Arbeitsgruppe der Deutschen Gesellschaft für Rheumatologie}

Bei einer Stichprobe von 10 Patienten wird die Lebensqualität über einen SLE Lebensqualitätsbogen/SF36-Bogen/SF-12-Bogen von der Helferin erfasst.

Einmal im Quartal wird der ECLAM erfasst. Eine Helferin erstellt einmal jährlich eine Auswertung.

Bei jedem Arztkontakt wird der SLEDAl erfasst und einmal jährlich über die Kerndokumentation des Rheumaforschungszentrums gemeldet und ausgewertet. Der Jahresbericht wird zunächst von den Ärzten besprochen und kurz in einer Teamsitzung vorstellt.

\section{Literatur}

Eckardt I, Sens B: Praxishandbuch Integrierte Behandlungspfade - Intersektorale und sektorale Prozesse professionell gestalten. Heidelberg: Economica 2006

Kopp I, Thole H, Selbmann HK, Ollenschläger G (Hrsg.): Deutsches Instrument zur methodischen Leitlinien-Bewertung (DELBI). Z ärztl Fortbild Qual. 2005; 99 (Sonderheft 8): 468-519 


\section{Praxisbeispiele}

Müller-Brodmann W, Edelmann E, Karger T: Rheumatologie. In: Kassenärztliche Bundesvereinigung, Berufsverband Deutscher Internisten, Birkner B, Diel F, Gibis B, Ludwig M (Hrsg.): „Qualitätsmanagement Innere Medizin“. Heidelberg: Springer 2007

Schlüter L, Roeder N, Schaff C: Behandlungspfade in der Kinder- und Jugendpsychiatrie. Münster: Schüling 2006

Wutzler U, Müller R: Einführung eines Qualitätsmanagements in den Praxen im Bereich der KV Südwürttemberg. Ärzteblatt Baden-Württemberg 2003; 1 